\title{
Paridad del poder adquisitivo en el tipo de cambio colombiano*
}

\author{
Purchasing power parity in the Colombian exchange rate
}

Paridade do poder de compra na taxa de câmbio colombiana

Received on May 30, 2017. Accepted on February 27, 2018

Oscar Hernán Cerquera Losada**

Colombia

Camilo Fabiam Gómez Segura***

Colombia

Cristian José Arias Barrera****

Colombia

, Para citar este artículo:

Cerquera Losada, Oscar Hernán;

Gómez Segura, Camilo Fabiam

y Arias Barrera, Cristian José

(diciembre, 2018). Paridad

del poder adquisitivo en el

tipo de cambio colombiano.

Ánfora, 25(45), 145-166. DOI:

https://doi.org/10.30854/anf

v25.45.2018.XXX Universidad

Autónoma de Manizales. ISSN

0121-6538.

\section{Resumen}

Objetivos: determinar el cumplimiento de la teoría de la Paridad del Poder Adquisitivo (PPA) en Colombia usando como patrón el tipo de cambio con el dólar estadounidense. Metodología: para comprobar si se cumple la PPA en Colombia, se utilizaron datos mensuales y trimestrales que van desde enero de 1959 a diciembre de 2015. Para ello, se modelizó el

\footnotetext{
* Este artículo es resultado de la investigación "Análisis del Poder de Paridad Adquisitivo en Colombia: una aplicación a diferentes divisas colombianas". Fue avalada por la Universidad Surcolombiana y se terminó en diciembre de 2016

** Magíster en Economía. Economista. Docente de la Universidad Surcolombiana. Joven Investigador e Innovador de Colciencias 2010-2011. Investigador del Observatorio Regional del Mercado de Trabajo del Huila, ORMET Huila. Integrante del grupo de investigación Iguaque. Correo: oscar.cerquera@usco.edu.co.

***Magíster en Economía. Economista. Docente de la Universidad Surcolombiana. Investigador del Observatorio Regional del Mercado de Trabajo del Huila, ORMET Huila. Integrante del grupo de investigación Iguaque. Correo: cafagose@hotmail.com

**** Economista. Analista económico de la Corporación Autónoma Regional del Alto Magdalena. Investigador económico del grupo de investigación Iguaque.
} 
comportamiento a largo plazo del tipo de cambio real, contrastando la presencia de raíces unitarias y cambios estructurales; además, se usó un modelo bivariado de cointegración. Resultados: se encontró que, para el caso de Colombia, no se cumplió la teoría de PPA, pues el peso y el dólar no están cointegrados. Por lo tanto, según la metodología de raíz unitaria no se valida la hipótesis de PPA en Colombia para el periodo 1959-2015. Todo parece indicar que el peso colombiano presenta poca relación con el dólar estadounidense. Conclusiones: el uso de índices de precios generales que incluyen bienes comercializables entre los países, las múltiples barreras al comercio internacional, la competencia imperfecta y las diferencias sociales, económicas, políticas y culturales entre los dos países, son aspectos importantes a la hora de explicar el incumplimiento de la PPA.

Palabras clave: Paridad del poder adquisitivo, Tipo de cambio real; Raíz unitaria, Cointegración.

\section{Abstract}

Objective: to determine the fulfillment of the purchasing power parity (PPP) theory in Colombia, the exchange rate with the US dollar using as a standard. Methodology: to check if the PPP in Colombia is achieved, monthly and quarterly data was used, which ran from January 1959 to December 2015. To do this, the long-term behavior from the real exchange rate was modeled, contrasting the unit roots presence and structural changes. In addition, a bivariate cointegration model was used. Results: it was found that, in the case of Colombia, the PPP theory was not fulfilled, since the peso and the dollar are not cointegrated. Therefore, according to the unitary root methodology, the PPP hypothesis in Colombia for the period 1959-2015 is not validated. Everything seems to indicate that the Colombian peso has little relation with the US dollar. Conclusions: the use of general price indices, which include tradable goods between countries, multiple barriers to international trade, imperfect competition and social, economic, political and cultural differences between both countries are important aspects when explaining the unfulfillment of the PPP.

Keywords: Purchasing power parity; Real exchange rate; Unitary root; Cointegration. 


\section{Resumo}

Objetivo: determinar o cumprimento da teoria de Paridade do Poder Aquisitivo (PPA) na Colômbia, utilizando como padrão a taxa de câmbio com o dólar norteamericano. Metodologia: para verificar se o PPA na Colômbia é cumprido, foram utilizados dados mensais e trimestrais, que vão de janeiro de 1959 a dezembro de 2015. Para fazer isso, o comportamento de longo prazo da taxa de câmbio real foi modelado, contrastando a presença de raízes unitárias e mudanças estruturais; Além disso, foi utilizado um modelo de cointegração bivariado. Resultados: constatou-se que, para o caso da Colômbia, a teoria do PPA não foi cumprida, uma vez que o peso e o dólar não estão cointegrados. Portanto, de acordo com a metodologia de raiz unitária, a hipótese do PPA na Colômbia para o período 1959-2015 não é validada. Tudo parece indicar que o peso colombiano tem pouca relação com o dólar americano. Conclusões: $\mathrm{o}$ uso de índices de preços gerais que incluem bens comercializáveis entre países, as múltiplas barreiras ao comércio internacional, a concorrência imperfeita e as diferenças sociais, econômicas, políticas e culturais entre os dois países são aspectos importantes no momento de explicar o descumprimento da PPA.

Palavras - chave: Paridade do poder aquisitivo, taxa de câmbio real; Raiz unitária, Cointegração. 


\section{Introducción}

Las primeras formulaciones sobre la teoría de la paridad del poder adquisitivo (PPA) se remontan al siglo XVI, planteado formalmente por Gustavo Cassel en 1918. Además, autores como Dornbusch (1987), Froot y Rogoff (1995) o Rogoff (1996) realizaron otros análisis de la PPA. Cassel (1918) se apoya en la Ley del Único precio para un producto que se comercializa en un mercado internacional integrado y competitivo (Rogoff, 1996). La Ley del Único Precio señala que en mercados competitivos (sin costos de transporte ni barreras al comercio), los productos idénticos vendidos en países deben tener el mismo precio si se mide en la misma moneda.

La paridad del poder adquisitivo es una de las medidas más adecuadas para comparar los niveles de vida, con ventajas sobre el producto interno bruto per cápita, puesto que toma en cuenta las variaciones de precios; este indicador elimina la ilusión monetaria ligada a la variación de los tipos de cambio, de tal manera que una apreciación o depreciación de una moneda no cambiará la paridad del poder adquisitivo de un país, puesto que sus habitantes reciben salarios y hacen compras en la misma moneda (Taylor, 2004).

Colombia ha empleado diferentes sistemas de tipo de cambio para regular el mercado de la moneda extranjera, desde el régimen de tipo de cambio múltiple para el periodo 1932-1967, hasta llegar al sistema actual de flotación vigente desde 1997.

El tipo de cambio múltiple se aplicó en Colombia con el objetivo fundamental de proteger las tenencias de oro del Banco de la República ante la crisis monetaria internacional, a partir de una intervención temporal en el mercado de divisas. A partir de 1967, con el Decreto-Ley 444, se estableció un régimen cambiario conocido como "crowling peg" o mini devaluaciones, como respuesta a las dramáticas devaluaciones y desequilibrios cambiarios que se presentaban con el sistema anterior.

Con el Crowling Peg se buscaba restablecer el nivel de competitividad de la economía colombiana; esto funcionaba igual que el sistema de tasa de cambio fijo, pero la diferencia es que bajo este sistema la tasa de cambio se fija a períodos mucho más cortos. Se adoptó un sistema de mini devaluaciones o "gota a gota", donde cada mes se presentaban pequeños ajustes sobre el tipo de cambio fijo, de acuerdo con la política monetaria planteada por el Banco de la República.

Según Wiesner (1978), el régimen de mini devaluaciones permitió que la tasa de cambio nominal se manejará de tal forma que se pretendía mantener una paridad adquisitiva efectiva teniendo en cuenta la inflación interna y externa, así como subsidios y gravámenes especiales. 
La década de los 80 fue un periodo en el que la tasa de cambio en Colombia se devaluó continuamente, debido a fenómenos como el aumento de las reservas internacionales en poder del Banco de la República a causa de los altos precios internacionales del café entre 1976 y 1978, el incremento en las exportaciones del grano en 1978 y a la deuda pública externa.

La constitución de Colombia de 1991 le otorgó total autonomía al Banco de la República para "mantener el poder adquisitivo de la moneda, (ante esto) el Emisor cambió su política económica hacia un objetivo fundamental: la reducción de la inflación” (Huertas et al., 2005). Dadas estas nuevas condiciones y los constantes choques en la balanza de pagos, el manejo de las reservas para disminuir la inflación y conservar el tipo de cambio fijo se vuelve inmanejable; ante esto se opta por un sistema más flexible que permita adquirir mayor autonomía monetaria. Es así como nace el sistema de bandas cambiarias en Colombia, el cual perdura desde enero de 1994 a septiembre de 1999.

A diferencia de los regímenes de tasa de cambio fijo o crowling peg, el sistema de bandas cambiarias conlleva fijar un techo y un piso para el precio de la divisa, los cuales fueron cambiados diariamente. Así, el techo y el piso de la banda se desplazaban paralelamente para que al final del año se alcanzará la meta de devaluación establecida que concordara con la meta esperada de inflación.

$\mathrm{El}$ sistema de bandas cambiarias fue muy criticada por los exportadores ya que en principio el peso se revaluó, aspecto perjudicial para los exportadores. Es así como en septiembre de 1998 la banda fue modificada como respuesta a las presiones especulativas de devaluación de los agentes económicos, después de un periodo largo en el que el precio del dólar estaba pegado al techo. En junio de 1999 la banda fue nuevamente modificada impulsada por la crisis económica del momento; estas variaciones en las bandas cambiarias de manera tan repetitiva le restaron credibilidad al régimen.

La poca credibilidad del sistema de bandas cambiarias, el creciente déficit fiscal del gobierno y la caída dramática de los flujos de capitales sumado a un retiro masivo de capitales producto de las crisis asiática y rusa, llevaron al Banco de la República a vender reservas y aumentar las tasas de interés para defender el régimen; esta situación se volvió insostenible ante la inminente recesión económica por lo que el Banco optó por eliminar este régimen, pero no antes de haber negociado un paquete de ajuste macroeconómico con el FMI que permitiera evitar un ataque especulativo contra el peso.

En septiembre de 1999 se adoptó un régimen de cambio flexible en el que la tasa de cambio flota libremente y es determinada por las fuerzas del mercado; este régimen esta imperante en la actualidad. Esencialmente, se acogió un régimen cambiario de flotación "sucia" que se caracteriza porque la oferta y la demanda pueden interactuar libremente mientras que el Banco de la República 
puede intervenir en el mercado cuando lo crea indispensable bajo unas reglas claras preestablecidas por el Banco; se estableció una nueva estructura de compra y venta de divisas.

Este trabajo tiene como objetivo estudiar la teoría de la paridad del poder de Colombia con respecto a los Estados Unidos durante el periodo de 1959 a 2015, usando datos de periodicidad mensual y trimestral. El contraste de la hipótesis de la PPA se realiza a través de dos métodos: i) contrastar la hipótesis de raíz unitaria y ii) análisis de cointegración propuesto por Engle y Granger (1987) y Johansen (1991). Por lo anterior, con este trabajo se busca responder la siguiente pregunta:

¿Se cumple la teoría de la paridad de poder adquisitivo de Colombia con respecto a Estados Unidos durante el periodo 1959-2015?

Estudiar el cumplimiento de la PPA es importante pues permite describir las inconsistencias entre las medidas económicas de los diferentes países. La PPA es una manera de analizar las diferencias en los niveles de precios entre los países, en este caso Colombia y Estados Unidos. En términos generales, el cumplimiento de la PPA permite deducir que los colombianos podrían comprar los bienes en Colombia al mismo precio que los comprarían en Estados Unidos.

\section{Metodología}

La idea fundamental de la teoría de la paridad del poder adquisitivo, es que el valor de cada moneda se determina por su poder adquisitivo como inversa del nivel de precios y el valor relativo de dos monedas o valor de cambio como la ratio de su poder adquisitivo; es decir, la PPA platea que el tipo de cambio entre dos monedas estará determinado por la relación entre los niveles de precios de estas dos economías.

Se deben distinguir dos aspectos dentro del concepto de la PPA, la PPA absoluta y la PPA relativa; la primera hace referencia al nivel absoluto de precios de un país y viene expresada en unidades monetarias del exterior por unidad monetaria del interior; la PPA relativa se refiere a las variaciones en los niveles de precios, no a su nivel absoluto; el modo de medir las variaciones es a través de los números índices a partir de un periodo base que se selecciona.

$\mathrm{Al}$ definir el valor de una moneda en términos de otra y los índices de precios nacionales $(\mathrm{P})$ e internacionales $\left(\mathrm{P}^{\wedge} *\right)$, podemos formular la hipótesis de PPA como:

$$
E_{t}=P_{t} / P_{t}^{*}
$$


Donde $E$ el tipo de cambio nominal $P$ y $P^{*}$ son los niveles de precios del país bajo estudio y el extranjero, respectivamente. Se desprende de la ecuación anterior que para que se cumpla la PPA se requiere que el tipo de cambio esté determinado por la ratio entre los precios relativos.

Si la PPA se cumple, resultaría lógico esperar que el poder adquisitivo entre ambas economías permanezca constante a lo largo del tiempo. Esto es que $P_{t} / P_{t}^{*}=k$ para todo $i$, entonces; $E={ }^{P} / P_{t}^{*}=k$ Ahora bien, si se plantea que $S$, el tipo de cambio real, refleja los precios relativos entre las dos economías medidos en la misma moneda, resulta que;

$$
S_{t}=E_{t} *\left[P_{t} / P_{t}^{*}\right]
$$

Si el valor obtenido por $S$ es igual a la unidad, se dice que se cumple la PPA en su versión absoluta.

Reordenando la ecuación (2) y transformando sus variables en logaritmos, se puede expresar la condición de cumplimiento de la PPA como:

$$
e_{t}=\alpha+\lambda\left(p_{t}-p_{t}^{*}\right)+s_{t}
$$

Donde $\alpha$ es la constante que reflejaría el impacto de los costos de transporte y las rigideces asociadas al comercio internacional, $e_{t}, p_{t} y p_{t}{ }^{*}$ son los logaritmos naturales del tipo de cambio nominal, del índice de precios nacionales y del índice de precios del país extranjero, respectivamente. Por último, $s \_t$ es el tipo de cambio real expresado en logaritmos naturales.

Teniendo claro el concepto de la PPA, el primer paso a realizar es la validación de la hipótesis de la PPA mediante la contrastación de estacionariedad de la serie tipo cambio de cambio real a partir de las pruebas ADF (Augmented Dickey-Fuller), Phillips-Perrón y ERS (Elliot, Rothenberg, and Stock Point Optimal)'aㅣ además se aplicó la prueba de Zivot y Andrews (1992), implementada por

1 La prueba ERS parte de la versión Dickey-Fuller estimada a partir de mínimos cuadrados generalizados. 
Trujillo (2004) en Eviews para corroborar la existencia de quiebre estructural en la serie estudiada.

Previo a la verificación de existencia de un vector de cointegración a través de la metodología de dos etapas propuesta por Engle y Granger (1987), se procedió a la contrastación del grado de integración de las dos variables, para luego verificar la cointegración; así mismo se verificó la condición de cointegración con la metodología desarrollada por Johansen (1991) a partir de un modelo de vectores autorregresivos VAR.

Para este estudio se utilizaron datos mensuales y trimestrales que van desde 1959:1 a 2015:12. En todos los casos los datos provienen del Departamento Nacional de Estadística (DANE), U.S. Bureau of Labor Statistics y Banco de la República. Las variables son: el tipo de cambio nominal (TCN) expresado por la cantidad de moneda colombiana necesaria para adquirir una unidad de moneda estadounidense; tipo de cambio real (TCR) expresado como el tipo de cambio nominal ajustado por diferencias en el nivel de precios o costos entre los países; los índices de precios al consumidor de Colombia (IPCCol) y los Estados Unidos (IPCEEUU). Todas estas variables están expresan en sus valores promedios para cada periodo y en algunos casos transformadas a logaritmos.

\section{Resultados}

En este apartado se contrasta el cumplimiento de la PPA para Colombia a través las pruebas de raíces unitarias y cambios estructurales. Una vez comprobada el cumplimiento o no de la teoría, se utiliza un modelo bivariado de cointegración para determinar si las variables utilizadas en la PPA presentan algún nivel de relación.

\section{Análisis de Raíz Unitaria}

La primera metodología desarrollada para probar la hipótesis de PPA es la de raíz unitaria sobre el tipo de cambio real. Por lo tanto, se calculó el tipo de cambio real con base en la siguiente formulación:

$$
T C R=T C N *\left[\frac{I P C^{U S A}}{I P C^{C o l}}\right]
$$


La gráfica 1 muestra el tipo de cambio real de Colombia con periodicidad trimestral y mensual.

Gráfica 1. Tipo de Cambio Real de Colombia 1959-2015
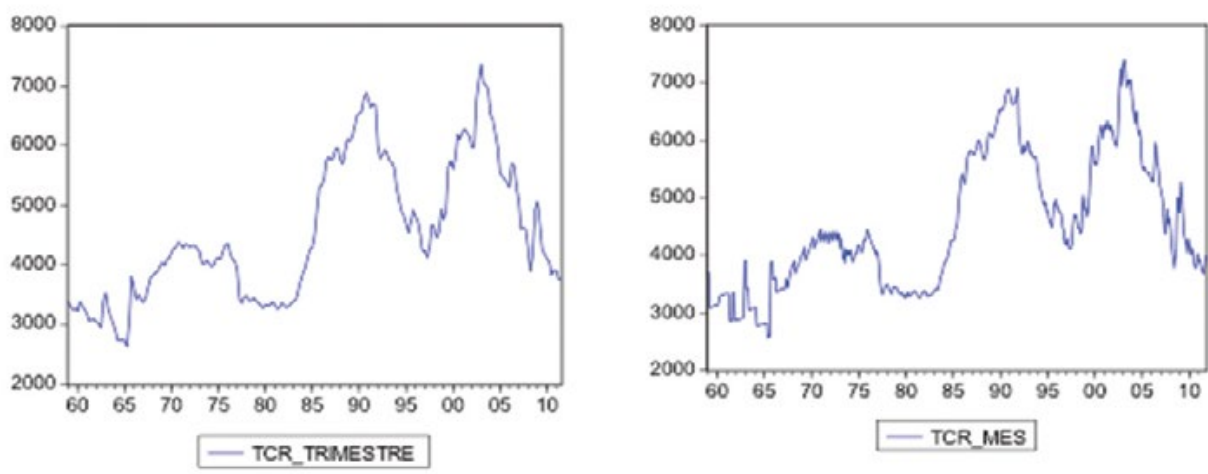

Fuente: elaboración propia

Este periodo de estudio se caracteriza por constantes fluctuaciones, explicadas básicamente por los cambios en regímenes de tipo de cambio analizados y por las condiciones económicas de la época, especialmente por la apertura económica de 1990.

La gráfica, además permite determinar si la serie tiene intercepto, tendencia o ninguna de las características de las series de tiempo; esto es útil para establecer el tipo de modelo cuando se realiza la prueba de raíz unitaria. Claramente la serie presenta intercepto, pero no existe evidencia suficiente para afirmar que el TCR tiene tendencia determinística, ya que la serie no parece girar alrededor de su media (\$4.535); de modo que esto permite concluir la pertinencia de incluir intercepto en el modelo.

Para realizar las pruebas de raíz unitaria se utilizaron los siguientes criterios de información: con la prueba $\mathrm{ADF}$ se utilizó el criterio de información de Schwarz; para la prueba de Phillips-Perrón se usó el criterio de Barlett-Kernel y como método de selección de ancho de banda el de Newey-West; y finalmente en la prueba ERS el criterio utilizado fue AR spectral OLS y el criterio de información de Schwarz. Los resultados se muestran en los siguientes cuadros. 
Cuadro 1. Prueba ADF para el TCR de Colombia 1959-2015

\begin{tabular}{c|c|c|c|c} 
Periodo & Ho= Raíz Unitaria & t-Statistic & P-Value & No. de Lags \\
\hline RER Month & Do not reject & -1.805133 & 0.3781 & 1 \\
\hline RER Quarter & Do not reject & $-1,81752$ & 0.3713 & 3
\end{tabular}

Fuente: elaboración propia

La prueba de Dickey-Fuller aumentada (ADF) plantea como hipótesis nula que existe una raíz unitaria para un cierto nivel de confianza (1\%, 5\%, o $10 \%)$. Por lo anterior, mientras más grande sea el t-statistic en valor absoluto, el P-value será más pequeño y tendrá mayor probabilidad de caer por debajo del nivel de significancia ${ }^{2}$ que permitan rechazar la hipótesis nula de raíz unitaria. En este caso, dado que el P-value es mayor que 0.05, se concluye que con la prueba de raíz unitaria $\mathrm{ADF}$, no existe suficiente evidencia para rechazar la hipótesis nula de raíz unitaria.

Cuadro 2. Prueba Phillips-Perrón para el TCR de Colombia 1959-2015

\begin{tabular}{c|c|c|c|c} 
Period & Ho = Unitary Root & Adj.t-Stat & P-Value & Bandwidth \\
\hline RER Month & Do not reject & -1.653432 & 0.4546 & 4 \\
\hline RER Quarter & Do not reject & -1.682069 & 0.4389 & 7
\end{tabular}

Fuente: elaboración propia

La prueba de raíz unitaria desarrollada por Phillips-Perron (1988), es una de las primeras pruebas en incorporar cambios estructurales en las series a lo largo del tiempo. En la hipótesis nula se plantea que no considera cambios estructurales en la serie de tiempo en estudio. Mientras más pequeño sea el Adj. t-Stat en valor absoluto, el P-value será más pequeño y tendrá mayor probabilidad de rechazar la hipótesis nula. En este caso, de nuevo el P-Value tanto para la serie mensual como trimestral es mayor que 0.05 , por lo que no existe evidencia empírica de rechazar la hipótesis nula.

2 Los niveles de significancia van de 1\% a 10\%. La mayoría de los teóricos coinciden en afirmar que un 5\% de nivel de significancia es adecuado. 
Cuadro 3. Prueba ERS para el TCR de Colombia 1959-2015

\begin{tabular}{c|c|c|c|c} 
Periodo & Ho= Raíz Unitaria & t-Statistic & Critical Values & No. de Lags \\
\hline \multirow{2}{*}{ TCR Mes } & Do not reject & 5,767246 & 1.9900 & \multirow{2}{*}{1} \\
& & & 3.2600 & \\
\cline { 3 - 3 } TCR Trimestre & Do not reject & 7.122928 & 3.17800 & \\
& \multicolumn{2}{|c}{ Fuente: elaboración propia }
\end{tabular}

Como se observa en los tres cuadros anteriores, no existe evidencia empírica para rechazar la hipótesis nula de raíz unitaria (en todos los casos se rechaza claramente que sean $I(0)$ y se comprueban que son $I(1)$, o su primera diferencia es estacionaria), por lo tanto, utilizando la metodología de raíz unitaria, no se valida la hipótesis de PPA en Colombia para el periodo 1959-2015. Todo parece indicar que el peso colombiano presenta poca relación con el dólar estadounidense.

Los resultados muestran que no hay suficiente evidencia para diagnosticar tendencia determinística en la serie TCR. Además, tampoco hay tendencia temporal $^{3}$ por lo que podría pensarse que el comportamiento errático de la serie puede deberse a un quiebre estructural en el nivel de la variable. Este choque puede atribuirse al proceso de apertura económica de los años 90 y la adopción de un régimen cambiario. Para determinar si existe quiebre estructural, se implementó el procedimiento descrito por Zivot y Andrews (1992).

Los gráficos 2 y 3, muestran los posibles quiebres en tendencia y en media de la TCR con periodicidad mensual y trimestral respectivamente. Se concluye que la serie TCR con periodicidad mensual y trimestral no presenta evidencia de que los cambios estructurales considerados tengan efectos significativos en la serie, ya que la línea asociada al resultado de la prueba aplicada secuencialmente tanto para el quiebre en media (zivotm) como para el quiebre en tendencia (zivott) no cruzan el valor crítico (VCRITM, VCRITT).

3 Porque las gráficas no muestran ningún comportamiento tendencial a lo largo del tiempo. 
Gráfico 2. Análisis de Quiebre Estructural TCR de Colombia (mes)
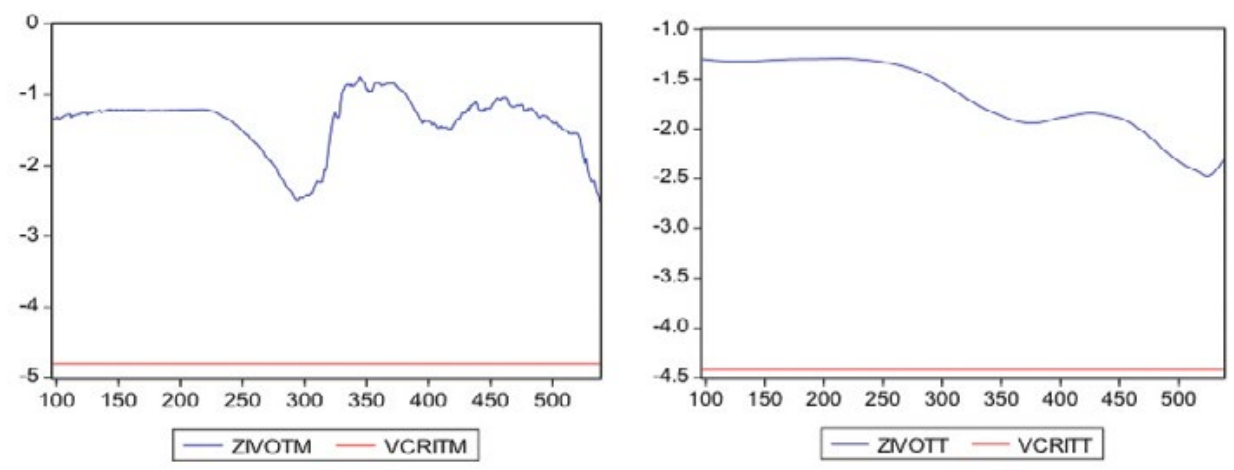

Fuente: elaboración propia

Gráfico 3. Análisis de Quiebre Estructural TCR de Colombia (trimestre)
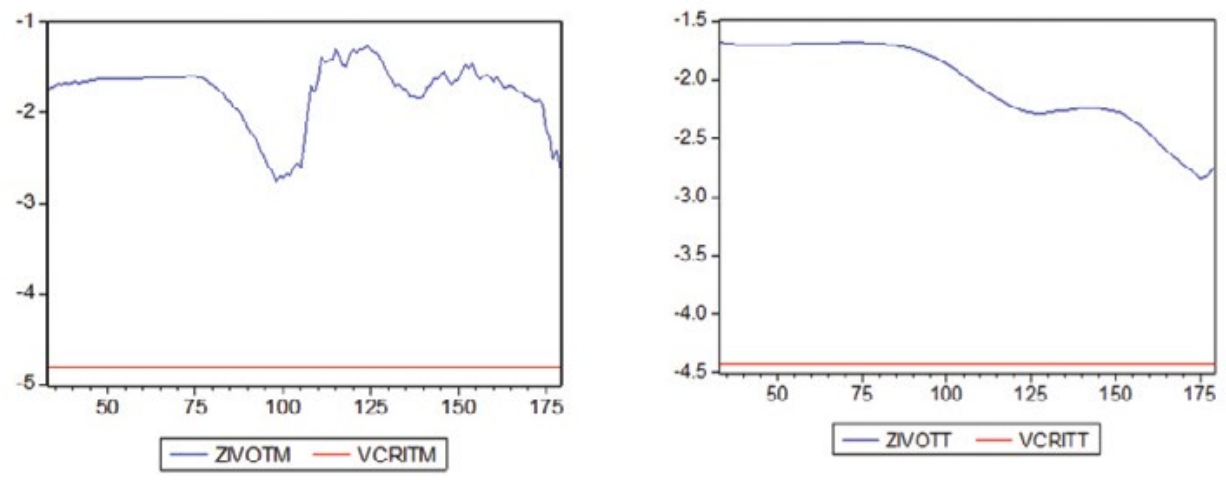

Fuente: elaboración propia

Por lo tanto, el comportamiento errático de la serie no se debe a quiebres estructurales sino a las características propias de la serie. Se sostiene que no existe evidencia empírica para rechazar la hipótesis nula de raíz unitaria, por lo que se mantiene la conclusión anterior de no estacionariedad en la serie TCR para Colombia.

Desde el punto de vista teórico, cuando una serie es no estacionaria, tiene raíces unitarias, por lo que realizar análisis econométrico con series no estacionarias puede llevar a estimar regresiones espurias, pues se concluiría que las series presentan un alto nivel de correlación cuando en realidad podría estar 
ocurriendo lo contrario. Para evitar este problema, se usa el análisis de cointegración que se utiliza cuando las series son no estacionarias.

\section{Análisis de cointegración}

Previo a la verificación de la condición de cointegración de las variables, es necesario identificar el orden de integración de las series a estudiar, el tipo de cambio nominal (TCN), y la diferencia entre IPC de Colombia e IPC de los Estados Unidos (IPC_COL_EEUU). Si las series son estacionarias se puede aplicar MCO sin perjuicio alguno sobre los coeficientes estimados, en otro caso se debe contrastar que la serie posea el mismo orden de integración para luego verificar si existe al menos una relación de cointegración a largo plazo entre las variables.

Los resultados de las pruebas de raíz unitaria ADF, Phillips-Perrón y ERS para las series de la ecuación (3) se muestran en el cuadro 4. Si las series presentan el mismo orden de integración se puede esperar que la combinación de éstas sea estacionaria; si esto ocurre existiría un vector de cointegración entre las series.

\section{Cuadro 4. Pruebas de Raíz Unitaria de TCN e IPC_COL_EEUU de Colombia}

\begin{tabular}{c|c|c|c|c|c|c}
\multirow{2}{*}{ Serie } & \multicolumn{2}{|c|}{ ADF } & \multicolumn{2}{c|}{ Phillips-Perrón } & \multicolumn{2}{c}{ ERS } \\
\cline { 2 - 7 } & Niveles & Prim. Dif. & Niveles & Prim. Dif. & Niveles & Prim. Dif. \\
\hline TCN Month & -0.1768 & $-15.4127^{* * *}$ & -0.2144 & $-15.8773^{* * *}$ & 67.2996 & $0.1199^{* * *}$ \\
\hline TCN Quarter & -0.2801 & $-9.8025^{* * *}$ & -0.3311 & $-9.0532^{* * *}$ & 63.7894 & $0.1767^{* * *}$ \\
\hline $\begin{array}{c}\text { IPC_COL_USA } \\
\text { Month }\end{array}$ & -1.5745 & $-14.1038^{* * *}$ & -1.4191 & $-14.3992^{* * *}$ & 613.1231 & $0.1404^{* * *}$ \\
\hline $\begin{array}{c}\text { IPC_COL_USA } \\
\text { Quarter }\end{array}$ & -1.1348 & $-2.9150^{* *}$ & -1.2489 & $-9.5019^{* * *}$ & 103.2 & $2.5274^{* *}$ \\
\hline
\end{tabular}

${ }^{*}$ Significativo al $10 \%,{ }^{* *}$ Significativo al $5 \%,{ }^{* * *}$ Significativo al $1 \%$.

\section{Fuente: elaboración propia.}

De acuerdo con estos resultados, se puede aceptar que todas las series son integradas de orden $1 \mathrm{I}(1)$, dado que la condición de cointegración entre las series requiere que las series sean integradas del mismo orden y la diferencia resultante de la combinación lineal entre ellas produce una serie estacionaria, así, se puede avanzar en el procedimiento para determinar si existe una relación de cointegración en el modelo presentado de la ecuación (3). 
Para esto se utilizó la técnica de dos etapas desarrollada por Engle y Granger (1987) sobre la formulación (3), cuya estimación econométrica se expresada de la siguiente forma:

$$
T C N=c+\lambda\left(I P C_{t}^{C o l-E E U U}\right)+s_{t}
$$

Donde $T C N$ es el tipo de cambio nominal expresado en logaritmo neperiano, $I P C_{t}^{(\text {Col-EEUU) }}$ es la diferencia entre los logaritmos de los índices de precios al consumidor entre Colombia y los Estados Unidos ${ }^{4} s^{t}$ son los residuos de la regresión que se espera sea una serie ruido blanco, expresando en este caso el logaritmo del tipo de cambio real.

Los resultados de la estimación se presentan a continuación.

\section{Cuadro 5. Estimación Modelo de Engle y Granger}

\begin{tabular}{c|c|c|c|c|c|c|c}
\multirow{2}{*}{ Periodicidad } & \multirow{2}{*}{ N. Obs. } & \multicolumn{2}{|c|}{ Coeficiente } & \multicolumn{2}{c|}{ Standard Error } & \multicolumn{2}{c}{ Prob. } \\
\cline { 3 - 8 } & & C & $\wedge$ & C & $\wedge$ & C & $\Lambda$ \\
\hline Monthly & 672 & 8.689215 & 1.090532 & 0.014569 & 0.00377 & 0 & 0.000 \\
\hline Quarterly & 224 & 8.689177 & 1.08987 & 0.025006 & 0.006462 & 0 & 0.000
\end{tabular}

Fuente: elaboración propia.

A fin de verificar la condición de cointegración de las variables, se debe realizar una prueba de raíz unitaria a los residuos estimados de la regresión. Si los residuos son I $(0)$ se comprueba que, de acuerdo a la metodología de Engle y Granger, hay una relación de cointegración entre el Tipo de Cambio Nominal y las diferencias de los índices de precio de Colombia y los Estados Unidos. Se utilizó para tales efectos la prueba Augmented Dickey-Fuller (dado que en pruebas de raíz unitaria sobre los residuos de una regresión no es apropiado incluir tendencia ni intercepto). Los resultados se presentan a continuación.

4 Las variables se expresan en logaritmos, dado que mediante esta transformación se linealizan las relaciones, simplificando los cálculos y las conclusiones que se derivan. 
Cuadro 6. Prueba ADF de los Residuos del Modelo de Engle y Granger

\begin{tabular}{c|c|c|c|c|c} 
Periodicidad & $\begin{array}{c}\text { t-Statistic } \\
\text { ADF }\end{array}$ & $\begin{array}{c}\text { Valor Critico } \\
\text { a } 5 \%^{5}\end{array}$ & Bandwidth & Jarque-Bera & Media \\
\hline Per month & -2.404838 & $-3,350$ & 3 & 6.054308 & $-7.33 \mathrm{e}-16$ \\
\hline Per quarter & $-1,877273$ & $-3,368$ & 1 & 19.68649 & $7.78 \mathrm{e}-16$
\end{tabular}

elaboración propia. ${ }^{*}$ Significativo al $10 \%,{ }^{* *}$ Significativo al 5\%, ${ }^{* * *}$ Significativo al $1 \%$.

Fuente: elaboración propia.

Los valores empíricos obtenidos (-2.404 para el caso mensual, y -1.877 para el caso trimestral) de la prueba ADF sobre los residuos de la regresión, contrastado con el valor -3.35 y -3.36 para los casos mensual y trimestral respectivamente, no permite rechazar la hipótesis nula de raíz unitaria en los residuos; es decir, confirman el carácter de no estacionariedad de la relación de cointegración. Por lo tanto, los residuos no son $\mathrm{I}(\mathrm{O})$, por lo que no existe una relación de cointegración entre el tipo de cambio nominal y las diferencias de los índices de precio de Colombia y los Estados Unidos.

\section{Gráfico 4. Normalidad de los Residuos, mensual y trimestral}
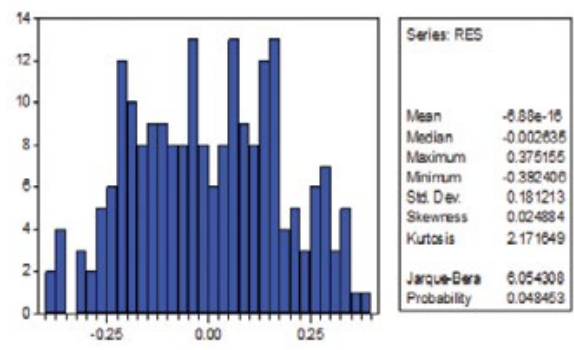

Fuente: elaboración propia.

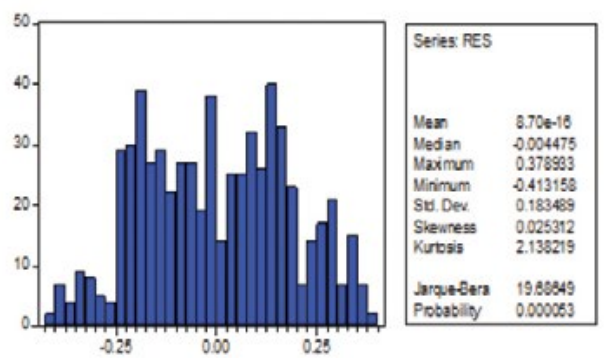

$$
\text { (1) }
$$


residuos, por lo tanto, se concluye que éstos no se comportan de forma normal. "La normalidad exacta de los estimadores MCO depende crucialmente de la normalidad de la distribución del error, u, en la población.

Si los errores $u_{1}, u_{2}, \ldots, u_{n}$ proviniesen de muestras aleatorias de una distribución distinta a una normal, los $\widehat{B}_{j}$ no estarían distribuidos como una normal; esto significa que los estadísticos t no tendrían distribución $t$ y que los estadísticos $F$ no tendrían distribuciones $F$, y esto es un problema serio. Estos resultados permiten concluir que los estimadores no son eficientes y no se puede decir que su distribución muestral es normal; por eso, la estimación de la variación del modelo se ve afectada. En cualquier caso, no se podría aceptar la hipótesis nula de los residuos igual a cero, condición importante para verificar el cumplimiento de la PPA.

Con el propósito de verificar la cointegración de las series se procede a desarrollar la metodología implementada por Johansen (1991) que permite verificar el número y la existencia probable de algún parámetro de cointegración; el primer paso, fue estimar el modelo VAR tomando como variables endógenas las series integradas de orden (1) (Tipo de cambio nominal y la diferencia entre el IPC de Colombia y de los Estados Unidos) y como variables endógenas la constante, para calcular la ordenada del origen.

La literatura recomienda que cuando se trabaje con datos trimestrales se debe tomar de 4 a 6 retardos; por lo tanto, el número de Lag Intervals for Endogeneous usado es de 1 a 6 , lo que indica 6 retardos, que van desde el 1 hasta el 6; para el caso de los datos mensuales se tomaron 16 retardos. Estimado el VAR, determinados el Lag Order Selection Criteria, bajo los criterios de Schwarz y Hannan-Quinn para los datos trimestrales (5 lags), y sequential modified LR, final prediction error y Akaike para los datos mensuales (19 lags).

El paso a seguir fue realizar la prueba de cointegración de Johansen para todos los tipos de modelos y con el número de rezagos establecidos, para determinar el número de relaciones de cointegración entre las series.

Cuadro 7. Prueba de Cointegración de Johansen

MONTHLY DATA

\begin{tabular}{c|c|c|c|c|c}
\hline Data Trend: & None & None & Linear & Linear & Quadratic \\
\hline Test Type & No Intercept & Intercept & Intercept & Intercept & Intercept \\
& No Trend & No Trend & No Trend & Trend & Trend \\
Trace & 0 & 0 & 0 & 0 & 0 \\
Max-Eig & 0 & 0 & 0 & 0 & 0 \\
\hline
\end{tabular}

* Critical values based on MacKinnon-Haug-Michelis (1999)

QUARTERLY DATA 


\begin{tabular}{c|c|c|c|c|c}
\hline Data Trend: & None & None & Linear & Linear & Quadratic \\
Test Type & No Intercept & Intercept & Intercept & Intercept & Intercept \\
& No Trend & No Trend & No Trend & Trend & Trend \\
Trace & 0 & 0 & 0 & 0 & 0 \\
Max-Eig & 0 & 0 & 0 & 0 & 0 \\
\hline
\end{tabular}

${ }^{*}$ Critical values based on MacKinnon-Haug-Michelis (1999)

Fuente: elaboración propia.

Los resultados muestran que no existe relación de cointegración de largo plazo entre las variables tipo de cambio nominal de Colombia y la diferencia del índice de precios al consumidor entre Colombia y los Estados Unidos, ambas series expresadas en logaritmos. Esto implica que no se cumple la hipótesis de la PPA para Colombia en el periodo 1959-2015. Estos resultados confirman lo encontrado con el método de raíz unitaria y la metodología de Engle y Granger (1987). En el siguiente apartado, se formulan algunas razones por las cuales no se cumplió la teoría del poder adquisitivo entre Colombia y Estados Unidos durante el periodo de referencia.

\section{Incumplimiento de la PPA}

El incumplimiento de la PPA en Colombia para el periodo estimado no quiere decir que el trabajo está perdido. La literatura sustenta muchas razones por las cuales no se valida la hipótesis de la PPA.

Balassa (1964) y Samuelson (1964) dieron separadamente una explicación del menor precio relativo de los bienes no comercializables en los países pobres, que es conocida como el “modelo Balassa-Samuelson”. Básicamente, este modelo destaca la diferencia en productividad entre los sectores de bienes comercializables y no comercializables en los distintos países y su efecto sobre los tipos de cambio reales.

Tales autores parten del hecho de que la productividad del trabajo es mayor en los países ricos que en los pobres, siendo esta diferenciación en productividad especialmente significativa en los bienes comercializables, al tiempo que los salarios se suponen iguales en el sector de bienes comercializables y en el de bienes no comercializables, positivamente relacionados con la productividad, aunque los salarios son los mismos en las dos industrias en cada una de las dos economías, la productividad es más elevada en el sector de bienes comercializables de la economía rica que en el país pobre, lo que eleva los salarios en el sector de bienes no comercializables, aunque no sea más eficiente que el país pobre en este sector; por tanto, utilizando el tipo de cambio para medir los precios de los bie- 
nes no comercializables, éstos son más elevados en los países desarrollados que en los países en desarrollo (Camarero, 1994).

La elección del índice apropiado para probar el cumplimiento de la PPA ha dado lugar a abundante literatura sobre el tema. Hakkio (1984) sostiene que la PPA puede fallar en presencia de perturbaciones de carácter real si se utilizan índices de carácter agregado en lugar de un índice de productos comercializables, dado que los bienes nacionales y extranjeros pueden ser sustitutos imperfectos, por lo que una perturbación real puede cambiar la demanda relativa de estos productos, precisando una modificación del tipo de cambio real. Algunos autores consideran que la utilización del IPC para medir la PPA puede introducir un sesgo y no se recomienda su empleo (Thygessen, 1978).

Según Cassel (1918), una de las condiciones necesarias para el cumplimiento de la PPA absoluta era la ausencia de barreras al libre comercio y costos de transporte; sin embargo, es evidente que ambos existen y que las restricciones al comercio han ido cambiando a lo largo de los años, haciendo difícil su medición dada su menor transparencia, pero afectando significativamente a los intercambios internacionales (Camarero, 1994). Es por eso que entre países vecinos y con elevado comercio se han obtenido tradicionalmente mejores resultados. Estados Unidos es el principal socio comercial para Colombia, pero lo contrario no ocurre. Además, el distanciamiento geográfico también es un factor en contra de la PPA, los costos de transporte, la cultura del consumo y demás aspectos sociales, económicos y políticos favorecen el cumplimiento de la PPA.

Frenkel (1981) llega a resultados interesantes al comparar los países europeos más importantes con los Estados Unidos; por ejemplo, que la PPA se cumple más frecuentemente entre países cercanos geográficamente y con alto nivel de intercambios. Miller (1984) también atribuye los mejores resultados entre los países europeos a los menores costos de transporte entre ellos y, en especial, al elemento institucional constituido por la Comunidad Económica Europea, con la consiguiente mayor integración comercial, financiera y monetaria.

Otra de las razones del incumplimiento de la PPA se basa en utilizar el dólar como moneda de referencia; esto se demuestra en numerosos trabajos desarrollados sobre el tema. Trozano (1992) realiza la contrastación de la PPA con varios métodos econométricos no encontrando diferencias entre ellos: las pruebas en las que participa el dólar dan peores resultados que los de otras monedas, como las europeas o el Yen.

La PPA se basa en la comparación de las mismas cestas de productos en dos países; de ahí que un importante problema que se plantee, desde el punto de vista estadístico, es que cada país asigna distintas ponderaciones a las diferentes categorías de bienes y servicios al elaborar los índices de precios (Camarero, 1994). 
Según Dombusch (1976), en un mundo en el que los mercados de capitales se hallan muy integrados y los mercados de bienes muestran ajustes lentos en los precios, existen sustanciales desviaciones de los tipos de cambio respecto a la PPA. La idea básica del modelo del overshooting es que, en el corto plazo, los precios de los bienes, tanto en la economía doméstica como en el exterior, pueden considerarse fijos, mientras que el tipo de cambio se ajusta rápidamente a la nueva información disponible y a cambios en la política económica. En ese caso, las variaciones del tipo de cambio pueden ser sustanciales y alejarse de la PPA durante periodos de tiempo prolongados. Según Frenkel (1981), los tipos de cambio y los niveles de precios no son comparables. El tipo de cambio puede considerarse el precio de un activo, sobre el cual influyen de manera decisiva las expectativas sobre el futuro, lo que da lugar a la elevada volatilidad de los tipos de cambio en épocas de incertidumbre.

\section{Conclusiones}

En este trabajo se probó la hipótesis de la Parida del Poder Adquisitivo entre la economía colombiana y la estadounidense, con las series mensuales y trimestrales para el periodo entre enero de 1959 y diciembre de 2015. Para ello, en base a la bibliografía más relevante sobre la materia, se probó la PPA mediante dos metodologías, contrastando la presencia de raíces unitarias y cambios estructurales, y con un modelo econométrico bivariado de cointegración.

Se pudo constatar que, a través de la prueba de raíces unitarias, el tipo de cambio real no es estacionario; por lo tanto, bajo esta metodología no se valida el cumplimiento de la PPA.

Con el modelo bivariado de Cointegración, se pudo verificar que las series son I(1) por medio de la prueba ADF (Augmented Dickey-Fuller), Phillips-Perrón y ERS (Elliot, Rothenberg, and Stock Point Optimal). A continuación, se analizó la posibilidad de cointegración utilizando la metodología de dos etapas de Engle y Granger (1987), con la cual se verificó que los residuos no son estacionarios, y además carecen de normalidad, por lo que se pudo verificar el incumplimiento de la PPA. Además, se implementó la metodología desarrollada por Johansen aplicado un modelo VAR, con la cual se pudo constatar que no existe ninguna relación de cointegración entre las variables analizadas.

Por lo tanto, y teniendo en cuenta los resultados de las dos metodologías implementadas, se concluye que no se acepta el cumplimiento de la hipótesis de la PPA para la economía colombiana en relación con la economía estadounidense para el período estudiado. 
Las razones del incumplimiento de la PPA son muchas, pero la literatura resalta especialmente el uso de índices de precios generales que incluyen bienes comercializables como no comerciales; las múltiples barreras al comercio internacional y la competencia imperfecta contradicen los supuestos necesarios para el cumplimiento de la PPA, además las diferencias sociales, económicas, políticas y culturales entre los dos países, son aspectos importantes a la hora de explicar el incumplimiento de la PPA.

Finalmente, desde el punto de vista estadístico, se concluye que cada país asigna distintas ponderaciones a las diferentes categorías de bienes y servicios al elaborar los índices de precios, dependiendo de las preferencias de los consumidores, las cuales varían mucho entre Colombia y los Estados Unidos, sólo por el hecho de que el último es un país desarrollado.

\section{References}

Balassa, B. (1964). The Purchasing-Power Parity Doctrine: A Reappraisal. Journal of Political Economy, 72(6), 584-96.

Cassel, G. (December, 1918). Abnormal Deviations in International Exchanges. Economic Journal, (28), 413-415.

Waiter, M. (1994). Empirical contributions of the Purchasing Power Parity. Journal of Applied Economics. vol.II. Jaume I University .

Dornbusch , R. (1976). Expectations and Exchange Rate Dynamics. Journal of Political Economy, 84 (6), 1161-176.

Engle, R. \& Granger, W. J. (1987). Co-integration and Error Correction: Representation, Estimation and Testing. Econometrica, 55 (2), 251-76.

Frenkel J. A. (1981). The Collapse of Purchasing Power Parities During the 1970's. European Economic Review, 16, 145-165.

Froot, K. and Rogoff , K. (1995). Perspectives on PPP and Long-Run Real Exchange Rates. NBER Working Papers, (4952), 1-57. National Bureau of Economic Research, Inc. 
Hakkio C. S. (1984). A Re-Examination of Purchasing Power Parity. A multi-country and multi-period study .Journal of International Economics, 17, 265-277.

Orchards, C., M. Khalil, S. Olarte and Romero, J. V. (2005). Some considerations on the credit channel and the transmission of interest rates in Colombia. Drafts of Economy 351, 1-38.

Johansen, S. (1991). Estimation and Hypothesis Testing of Cointegration Vectors in Gaussian Vector Autoregressive Models. Econometrica, 59 (6), 1551-1580.

Miller S. (1984). Purchasing Power Parity and Relative Price Variability. Evidence from the 1970's. European Economic Review, 26, 353-367.

Phillips, PCB \& Perron, P. (1988). Testing for a Unit Root in Time Series Regression. Biometrika, 75, 335-346.

Samuelson, P. (1964). Theoretical Notes on Trade Problems. Review of Economics and Statistics, 46(2), 145-54.

Taylor, M. (2004). Is Official Exchange Rate Intervention Effective? Economica, $71(1), 1-12$.

Thygessen, N. (1978). Inflation and Exchange Rates. Evidence and policy guidelines for the European Community.Journal of International Economics, 8, 301-317.

Trozano M. (1992). Long-Run Purchasing Power Parity and Mean-Reversion in Real Exchange Rates: a Further Assessment. International Economy, XLV, $77-100$.

Trujillo, G. H. (2004). Zivot \& Andrews Sequential Test. Recuperado de www. Gestiopolis.com.

Rogoff, K. (nineteen ninety six). The Purchasing Power Parity Puzzle. Journal of Economic Literature, XXXIV, 647-668.

Wiesner, E. (1978). Devaluation and Adjustment Mechanisms in Colombia. Banking and Finance Magazine, (179), 77-127. 
Zivot, E. and Andrews, D. (1992). Further evidence on the great crash, the oil-price shock and the unit-root hypothesis. Journal of Business and Economic Statistics, (10), 251-270. 